\title{
A Study of the Discrete Electron-wave Interaction for the Passbands and Stopbands of TWT Slow-wave Systems
}

\author{
Victor A. Solntsev, Roman P. Koltunov, Dmitry S. Shabanov \\ Moscow State Institute of Electronics \& Mathematics \\ 3, Bol. Trekhsvyatitelsky per. Moscow, 109028, Russia \\ Phone: +7(495)916-8858, fax: +7(495)916-2807, e-mail: rt@miem.edu.ru
}

\begin{abstract}
The fourth power algebraic characteristic equation is obtained for the wave number of the individual electron waves that exist in periodic slow-wave systems(SWS) with discrete electron-wave interaction. The study of properties of these waves and the solution of the corresponding boundary-value problem have been performed. It has allowed to find some properties of electron-wave interaction which are different or are identical to the SWS with discrete interactions or "smooth" SWS, for example helical SWS.
\end{abstract}

Keywords: slow-wave system; electron beam; interaction; TWT; passband; stopband.

\section{Introduction}

The equations of the discrete electron-wave interaction in TWT with periodic slow-wave structures (SWS) of the type of a coupled cavities system or of a disk-loaded waveguide have been analyzed in $[1,2]$ and the interaction equations of small-signal theory have been obtained. Unlike in the previously known works, the equation of excitation of periodic waveguides with second-order finite differences [3] and the local interaction impedance instead of Pierce's coupling impedance are used in the suggested theory. It allows to get the unified description the SWS interaction of electrons with a full field of two waves - forward and backward - in both the passband and the stopband (Fig.1).

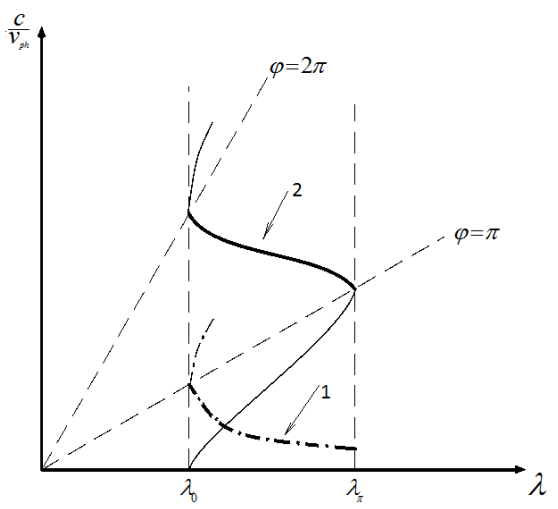

Figure 1. The dispersion characteristics for 1) helical SWS (- -, $\left.\varphi_{0}\right)$ and 2) coupled cavities system $\left(-, \varphi_{1}\right)$ in passband

In the small-signal theory equation for the electron beam RF current $\mathrm{J}$ has a well-known form, such as shown in $[1,2]$ :

$$
\frac{d^{2} J}{d z^{2}}-2 i h_{e} \frac{d J}{d z}+\left(\Gamma h_{p}^{2}-h_{e}^{2}\right) J=-i h_{p}^{2} S \omega \varepsilon_{0} \bar{E}
$$

where $h_{e}=\omega / v_{e}$ - electron wavenumber, $h_{p}=\omega_{p} / v_{e}$ - wavenumber of plasma, $\mathrm{S}$ - effective cross-sectional area of electron beam, $\sqrt{\tilde{A}}-$ reduction coefficient of plasma frequency $\omega_{p}$. In the interaction gaps on the electron beam operates the total electric field of direct and backward-waves $\quad \bar{E}=E_{s}+E_{-s}=E_{q}=-U_{q} / d$, where $d$-effective width of gap, $U_{\mathrm{q}}$ - the voltage at $q-\mathrm{m}$ gap. The voltage $U_{\mathrm{q}}$ defined beam current and must satisfy the equation excitation periodic SWS. This equation is the excitation finite-difference equation for quadripoles circuit describing periodic SWS (Fig.2).

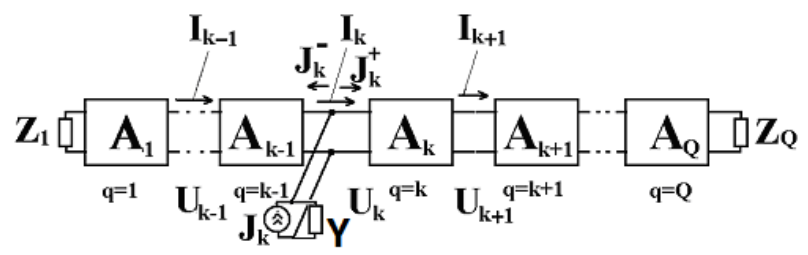

Figure 2. One section of periodic slow-wave system as circuit of quadripoles

$$
\Delta^{2} U_{q}+2 U_{q}\left(1-\cos \varphi_{s}\right)=i Z_{s} J_{q},
$$

where $Z_{s}=R_{s} L^{2} \sin \varphi_{s}$ - local interaction impedance, $R_{S}$ - specific coupling impedance, $L$ - the period of gap distance, $\varphi_{s}$ - the phase shift of the field over the period.

The current induced in the $q$ gap is determined from the expression

$$
J_{q}=\frac{1}{d} \int_{z_{q}^{-}}^{z_{q}^{+}} J(z) \bar{e}_{q}(z) d z=J_{0} \tilde{I}_{q} e^{i h_{e} z_{q}},
$$

where normalized induced current is

$$
\tilde{I}_{q}=I_{q} M-V_{q} C\left[\exp \left(i \frac{\theta}{2}\right)-M\right]+F_{q}^{m} C^{2} \theta Y(\theta),
$$

where $M=\sin \left(\frac{\theta}{2}\right) / \frac{\theta}{2}$ - interaction coefficient, $\theta$ - the gap transit angle, $Y(\theta)$ - complex conductance of electron gap, $C$ - the gain parameter, which is calculated using the local interaction impedance, $F_{q}^{m}, \mathrm{I}_{q}, V_{q}$ - normalized gap field, RF current and RF velocity of electron beam. 
Equations (1)-(4) are system of equations describing the discrete small-signal electron-wave interaction in passbands and stopbands.

\section{The properties of electron waves}

In this study, the fourth power algebraic characteristic equation is obtained for the wave number of the individual electron waves that exist in this system. The study of properties of these waves and the solution of the corresponding boundary-value problem have been performed. It has allowed to find some properties of electron-wave interaction which are different or are identical to the SWS with discrete interactions or "smooth" SWS.

In case of the SWS with the main spatial harmonic having the phase shift $\varphi_{0}$ at period $\mathrm{L}\left(0<\varphi_{0}<\pi\right)$, at $\varphi_{0} \rightarrow 0$ (ie. for decreasing of $\mathrm{L}$ ) the characteristic equation becomes the cubic equation, which coincides with the known equation of the Peirce's theory, obtained for the helical SWS. With the increasing $\varphi_{0}$ an effect of backward wave appears. It leads to a small change of the wave number already in the middle of the SWS passband at $\varphi_{0}=\pi / 2$. Increasing the phase further to the point of cut-off $\varphi_{0}=\pi$ leads to a substantial change in the electron waves wave numbers due to interaction of electrons with the spatial harmonic of SWS backward wave. There is a reactive attenuation after SWS cut-off point, so that $\varphi_{0}=\pi+i \cdot \operatorname{Im}\left(\varphi_{0}\right)$. However, gaining one electron waves remains and the gain band expands. The magnitude of expanding that band depends on the interaction parameters and can be calculated from the resulting characteristic equation for the each specific case (Fig. 3).

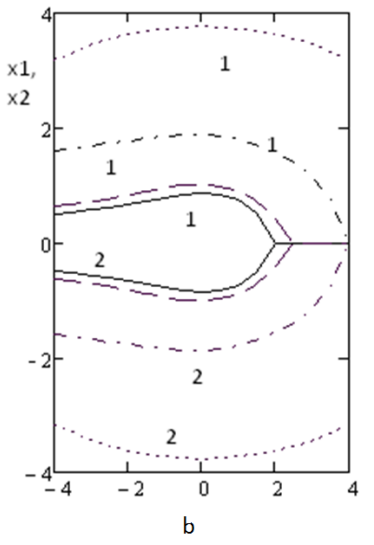

Figure 3. Roots $x_{1}=-x_{2}$ of the characteristic equation calculated for the case main spatial harmonic:

$\varphi_{\mathrm{s}} \rightarrow 0$ (-, smooth SWS); $\varphi_{s} \rightarrow \pi / 2(---) ; \varphi_{s} \rightarrow 0.9 \pi(--$ $-) ; \varphi_{\mathrm{s}} \rightarrow \pi(\ldots)$;

Similar results are obtained for using the first spatial harmonic as the working one, in the coupled cavities systems and similarly folded SWS, where $\varphi_{1}=\varphi_{0}+\pi$. However, the SWS remains as a periodic structure for $\varphi_{0} \rightarrow 0$ (ie. $\varphi_{1} \rightarrow \pi$ ), and it is then possible to gain in the stopband for $\varphi_{1}=\pi+i \cdot \operatorname{Im}\left(\varphi_{1}\right)$ as well.

We give examples of calculating the field distribution and the RF beam current along one section of passband or stopband sections of SWS, showing the possibility of increasing the RF current (and the field in some regime ) in the stopband sections.

The calculation was performed by solving the boundaryvalue problem with given reflection coefficients at the ends of sections and recurrent recalculation of the field and RF current from the gap to the gap without a representation as the sum of the electron waves in the system "periodic SWS-electron beam".

\section{Simulation the multisection TWT with stopband sections}

In addition to the study of electron waves in separate sections of SWS, the multisection TWT with stopband sections has been simulated. The methods of individual sections conjugation for the simulation were elaborated, and the distribution of RF currents and fields along the SWS were found. The resulting gain coefficient by small signal is close to the experimental data (Fig 4).

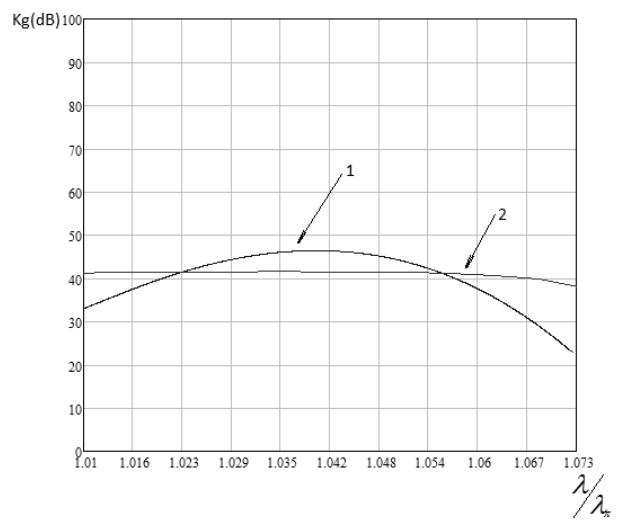

Figure 4. Calculated (1) and experimental (2) gain coefficient in passband, $\lambda_{\pi}$ according to stopsection cut-off.

This work was supported by the Russian Foundation for Basic Research (project number 10-02-00 859-a)

\section{References}

1. V.A.Solntsev and R.P. Koltunov. Analysis of the Equations of Discrete Electron-Wave Interaction and Electron-Beam Bunching in Periodic and Pseudoperiodic Slow-Wave Structures // Journal of Communications Technology and Electronics, 2008, Vol. 53, No. 6, pp. 700-713 Transl. from Radiotekhnika i Elektronika, 2008, Vol. 53, No. 6 pp. $738-751$.

2. V.A.Solntsev and R.P. Koltunov. A Generalized Linear Theory of the Discrete Electron-Wave Interaction in SlowWave Structures // Journal of Communications Technology and Electronics, 2008, Vol. 55, No. 11, pp. 1271-1284. Transl. from Radiotekhnika i Elektronika, 2010, Vol. 55, No. 11 pp. 1362-1374.

3. V. A. Solntsev and S. V. Mukhin, Radiotekh. Elektron.(Moscow) 36, 2161 (1991). 